\title{
Self Consistent Simulation of Dark Matter and Background
}

\section{Saptashwa Bhattacharyya*,Holger Motz $^{a}$,Shoji Torii ${ }^{b}$, Yoichi Asaoka $^{b}$,Yuko Okada}

\author{
ASE Waseda University \\ ${ }^{a}$ ICSEP Waseda University \\ ${ }^{b}$ RISE Waseda University \\ E-mail: saptashwaberuri.waseda.jp
}

\begin{abstract}
Future space based experiments such as CALET and DAMPE will measure the electron and positron cosmic-ray spectrum with better energy resolution and up to higher energy, making detection of small features in the spectrum, which might originate from Dark Matter annihilation or decay in the galactic halo, possible. For precise prediction of these features, the numerical cosmic ray propagation code GALPROP is used, and was extended to calculate the flux at Earth from different Dark Matter scenarios with any given injection spectrum. The results from GALPROP for both the cosmic-ray background spectrum and the component from Dark Matter annihilation are dependent on the bin size in energy used in the calculation, due to energy loss playing a major role in the propagation of electrons. A modification to partly compensate the influence of the discretization of the energy shifted particles has been implemented in the code. The effect of this improvement is demonstrated with examples of the expected spectra for the cosmic ray background in combination with the annihilation spectrum of LKP Dark Matter calculated at different energy binning.
\end{abstract}

The 34th International Cosmic Ray Conference,

30 July- 6 August, 2015

The Hague, The Netherlands

\footnotetext{
* Speaker.
} 


\section{Introduction}

As the first of a new generation of cosmic ray experiments capable of measuring directly the electron+positron in the $\mathrm{TeV}$ region, CALET (CALorimetric Electron Telescope) will be launched to the ISS until October of this year for its 5-year mission. CALET has the capability to take a sky survey of $e^{+}+e^{-}$and gamma rays in the energy range from $10 \mathrm{GeV}$ to more than $10 \mathrm{TeV}$ with an energy resolution of $2 \sim 3 \%$ [1] . To be on par with these new experimental possibilities, we aim to study cosmic ray (CR) propagation with equally high precision. We used the GALPROP code to study CR propagation and identified an energy binning dependence that arises from the calculation of radiative energy loss processes for electrons and positrons. We describe this effect and provide a method to reduce its influence on the propagated electron spectrum. By showing the influence of the binning dependence on the parameters of fitting the power law background spectrum together with flux from Dark Matter to the AMS-02 data, and how the modification reduces the differences, the benefit of this modified version of GALPROP for consistent calculation of both background and DM annihilation spectrum is explained.

\section{Cosmic Ray Propagation in GALPROP}

The version of GALPROP [2] this study is based on, is the latest public release (54.1.984), and in general a 3-dimensional spacial grid was used. As calculation of diffusive reacceleration and convection was switched off and only electrons (and protons for comparison) were studied, the transport equation given in [3], which GALPROP solves numerically to calculate propagation of charged CR particles, simplifies to the following diffusion equation:

$$
\frac{\partial \psi}{\partial t}=Q(\vec{r}, p)+\nabla \cdot\left(D_{x x} \nabla \psi\right)-\frac{\partial}{\partial p}(\dot{p} \psi)
$$

As described in [3], $\psi$ is the density of CR particles per unit momentum interval, $Q(\vec{r}, p)$ is the source term, $D_{x x}$ is the spatial diffusion coefficient and $\dot{p}$ is the momentum loss rate. The spatial diffusion coefficient is parametrized as $D_{x x}=D_{0} \beta\left(\frac{R}{R_{0}}\right)^{\delta}$ where $R$ is the rigidity of the cosmic ray

\begin{tabular}{|l|l|l|}
\hline Parameter & Value & Unit \\
\hline $\mathrm{Z}$ (height) $/ \Delta Z$ & $4 / 1$ & $\mathrm{kpc}$ \\
\hline $\mathrm{X} / \Delta X$ & $20 / 1$ & $\mathrm{kpc}$ \\
\hline $\mathrm{Y} / \Delta Y$ & $20 / 1$ & $\mathrm{kpc}$ \\
\hline$E_{\min }$ & $1.14 \times 10^{4}$ & $\mathrm{MeV}$ \\
\hline$E_{\max }$ & $1.0 \times 10^{7}$ & $\mathrm{MeV}$ \\
\hline$D_{00}$ (Diff. coeff.) & $5.70 \times 10^{28}$ & $\mathrm{~cm}^{2} \mathrm{sec}^{-1}$ \\
\hline$R_{0}$ (ref. rigidity for diff. coeff.) & 4 & $\mathrm{GV}$ \\
\hline$\delta$ (Diff. coeff. index) & 0.5 & \\
\hline$\gamma$ (electron injection index) & 2.80 & \\
\hline
\end{tabular}

Table 1: GALDEF file parameters used for CR propagation in GALPROP. 
particles and $\delta$ is the power law index above the reference rigidity $R_{0}$. As we focus on propagation of electrons, for which $p=E / c$, we will in the following refer to energy as equivalent of momentum. The energy grid in GALPROP is defined by minimum energy $\left(E_{\min }\right)$ and maximum energy $\left(E_{\text {max }}\right)$, as well as $E_{\text {factor }}$, which is the ratio of energy in successive grid points, i.e the energy of grid point $i$ is calculated as $E_{i}=E_{\text {min }}\left(E_{\text {factor }}\right)^{i}$. We assume each grid point to represent the cosmic ray particles inside a bin with lower boundary $E_{i(\text { low })}=E_{\text {min }}\left(E_{\text {factor }}\right)^{i-0.5}$ and upper boundary $E_{i(\text { high })}=E_{\text {min }}\left(E_{\text {factor }}\right)^{i+0.5}$. An overview of the values chosen for above parameters is given in Table 1 .

\subsection{Energy Binning Dependence of Electron Spectrum}

Cosmic rays lose energy during propagation by interaction with the interstellar medium, interstellar radiation field and the galactic magnetic field. For CR electrons inverse Compton scattering and synchrotron radiation are the main processes by which they lose energy. The corresponding terms constituting the momentum loss term $\left(\frac{\partial}{\partial p} \dot{p} \psi\right)$ are calculated in GALPROP beforehand for the fixed values of the energy grid, and stored in an array for use during calculation of the CR propagation. This causes the precision of the energy loss calculation for electrons to be dependent on the energy grid bin size. This is explained in Figure 1 by a simplified example. Considering a power law spectrum $E^{-3}$ and calculating the average energy of the particles given by $\bar{E}=\left(\int_{E_{n}}^{E_{n+1}} f(E) E d E\right) /\left(\int_{E_{n}}^{E_{n+1}} f(E) d E\right)$ inside one energy bin, it is shown that for fine energy

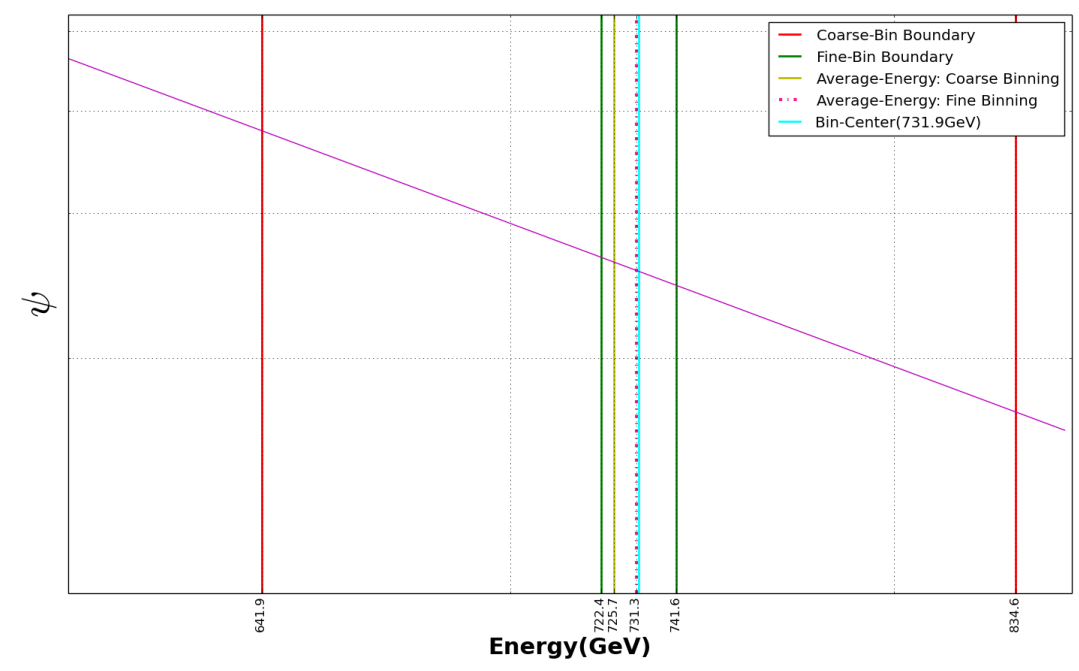

Figure 1: The magenta line represents a power law spectrum $\psi=E^{-3}$ inside the energy bin with bin center at $731.9 \mathrm{GeV}$ given by the cyan vertical line. The bin boundaries are described by the red lines for coarse binning $\left(E_{\text {factor }}: 1.3\right)$ and by the green lines for finer energy binning ( $E_{\text {factor }}: 1.03$ ). The average energy of the particles inside this bin is $725.7 \mathrm{GeV}$ for coarse binning (yellow line) compared to $731.3 \mathrm{GeV}$ for the finer binning. The deviation of the average energy is much smaller for fine binning $(0.6 \mathrm{GeV})$, than for coarse binning $(6.2 \mathrm{GeV})$. 


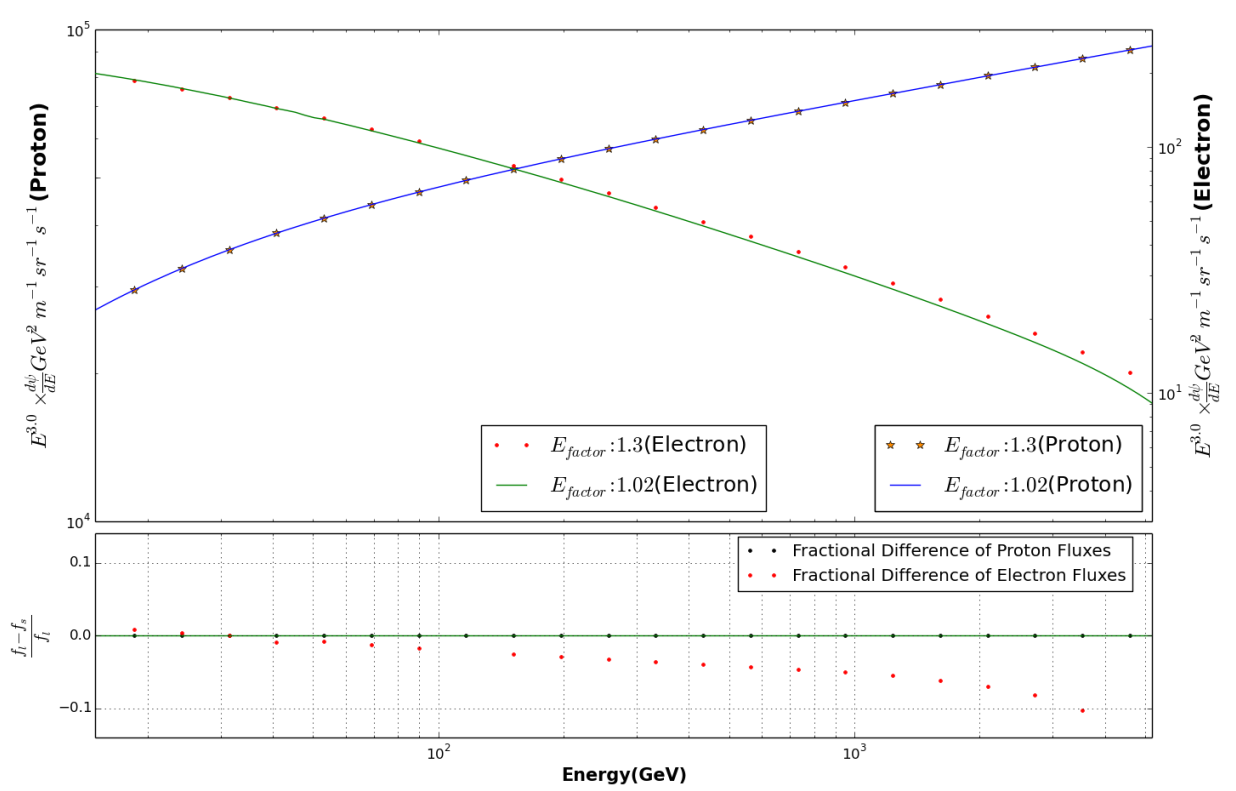

Figure 2: The figure shows electron spectrum and proton spectrum calculated at different energy binning (for electron, red dots $-E_{\text {factor }}: 1.3$, green line $-E_{\text {factor }}: 1.02$ and for proton, orange stars - $E_{\text {factor }}: 1.3$, blue line - $\left.E_{\text {factor }}: 1.02\right)$ in GALPROP. The lower panel shows the fractional difference of fluxes calculated with these two different energy binning for proton (black dots) and electron (red dots).

binning this average energy is very close to the energy grid point, while it is significantly shifted to lower energy for the coarse binning. Therefore the energy loss of electrons is more accurately taken into account for a fine energy binning calculation. The effect of this binning dependence is shown in Figure 2, which also demonstrates that the effect is not prominent for hadrons due to their much lower radiative energy loss compared to electrons. The power law index of the propagated primary electron spectrum decreases gradually with reduced $E_{\text {factor }}$, i.e. a finer energy binning.

The actual electron spectrum at a given time and point in space and therefore the average energy of the particles in a given bin is only known during the propagation calculation, where calculation of the energy loss terms at the required average energy for each case would cause prohibitive increase in calculation time. Therefore a method making use of the pre-calculated values was implemented to reduce the energy binning effect. The CR propagation equation is solved numerically by the Crank-Nicolson Method in GALPROP [3]. This method gives rise to a system of equations of the form:

$$
\frac{\alpha_{1}}{2} \psi_{i-1}^{t+\Delta t}+\left(1+\frac{\alpha_{2}}{2}\right) \psi_{i}^{t+\Delta t}-\frac{\alpha_{3}}{2} \psi_{i}^{t+\Delta t}=\frac{\alpha_{1}}{2} \psi_{i-1}^{t}+\left(1-\frac{\alpha_{2}}{2}\right) \psi_{i}^{t}+\frac{\alpha_{3}}{2} \psi_{i+1}^{t}+Q_{i} \Delta t
$$

$\psi$ and $\mathrm{Q}$ are defined as in equation 2.1. This equation is solved for $\psi_{i}^{t+\Delta t}$ to obtain the particle density $\psi$ after advancing time by $\Delta t$. Since the energy binning dependence arises from the energy loss term, we consider the components of $\alpha_{1}, \alpha_{2}, \alpha_{3}$ for the energy loss term, which are given for 
energy grid point $i$ by

$$
\alpha_{1 i}=0, \quad \alpha_{2 i}=\frac{\dot{p}_{i}}{p_{i}-p_{i+1}}, \quad \quad \alpha_{2 i}=\frac{-\dot{p}_{i+1}}{p_{i}-p_{i+1}}
$$

The average energy of particles in a bin doesn't coincide with the energy for which GALPROP calculates these coefficients. We try to reduce the effect by interpolation of these coefficients between grid point $i$ and grid point $i+1$ by a function of $\psi_{i}$ and $\psi_{i+1}$, the number density of cosmic ray particles at energy grid points $i$ and $i+1$. Among several tested interpolation procedures the best results could be achieved by defining $P=\left(\frac{\psi_{i}-\psi_{i+1}}{\psi_{i}}\right)^{2}$ and redefining $\alpha_{2 i}$ and $\alpha_{3 i}$ as

$$
\begin{aligned}
\alpha_{2 i} & =\frac{\dot{p}_{i}}{p_{i}-p_{i+1}} \times(1.0-P) \\
\alpha_{3 i} & =\frac{\dot{p}_{i+1}}{p_{i}-p_{i+1}} \times P
\end{aligned}
$$

By this modification the effect of binning on the propagated electron spectrum is reduced, as shown in Figure 3. Though a slight difference smaller than $2 \%$ remains, the strong increase of the discrepancy with energy is removed. This allows for reliable calculation of electron CR propagation with

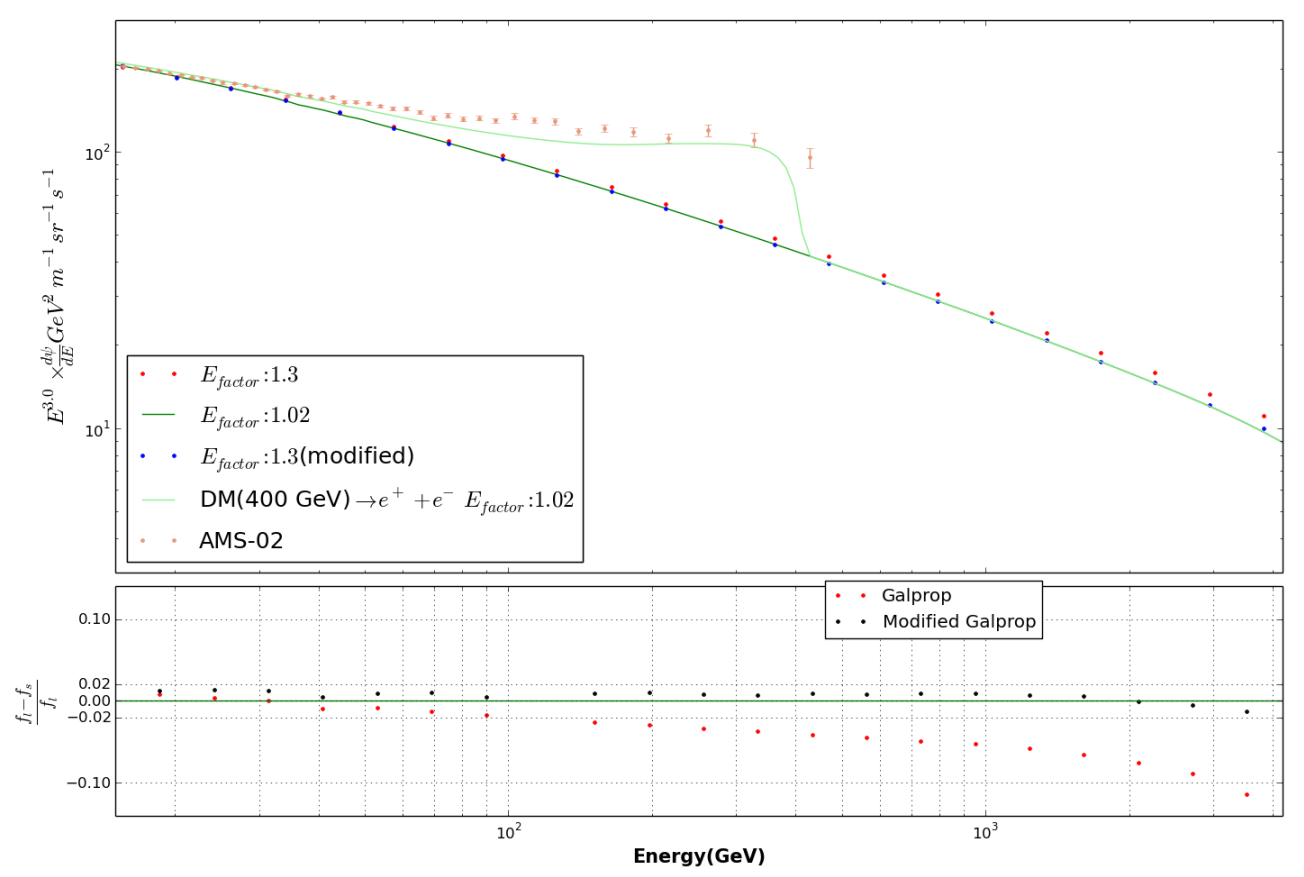

Figure 3: The effect of the modification in the CR spectrum is demonstrated in this figure. We plotted CR electron spectrum at two different energy binning $E_{\text {factor }}: 1.3$ (red dots), $E_{\text {factor }}: 1.02$ (green line). The modified GALPROP code was used to plot CR electron spectrum with $E_{\text {factor }}: 1.3$ (blue dots). The lower panel shows the fractional difference of electron fluxes calculated at two different energy binning ( $E_{\text {factor }}: 1.3$ and $\left.E_{\text {factor }}: 1.02\right)$ with (red dots) and without modification (black dots). 
a coarser energy binning, reducing memory usage and computation time compared to increasing the precision by choosing a smaller $E_{\text {factor }}$.

\section{Self Consistent Simulation of Background and DM}

The modified GALPROP code helps to treat background and Dark Matter annihilation spectrum consistently, which we demonstrate on the example of 1 TeV LKP Dark Matter. The DM annihilation spectra of the component annihilation channels

$\left(20 \% e^{+} e^{-}, 20 \% \mu^{+} \mu^{-}, 20 \% \tau^{+} \tau^{-}, 11 \% u+\bar{u}, 11 \% c+\bar{c}, 11 \% t+\bar{t}, 2 \% h+\bar{h}\right)$ at production are taken from the publicly available PPPC4 [4] tables. The source term for the DM annihilation has the form

$$
Q=\frac{1}{2}\langle\sigma v\rangle\left(\frac{\rho}{M_{D M}}\right)^{2} \frac{d N}{d E}
$$

where $\langle\sigma v\rangle$ is the velocity averaged annihilation x-section, $\rho$ is the DM density, $M_{D M}$ is the Dark Matter mass, and $\frac{d N}{d E}$ is the spectrum of the CR particles produced per annihilation. We choose the DM distribution to follow the NFW halo profile [5], normalized to a local density of $\rho_{0}=0.3 \mathrm{GeV}$. Both background CR spectrum and DM annihilation spectrum are propagated in GALPROP using the propagation parameters listed in Table 1. The propagation results are compared and fitted to recent AMS -02 results for the electron spectrum [6]. The flux $\phi_{D M}$ from Dark Matter annihilation is scaled by a boost factor $x$ and the power law index of the propagated background spectrum $\phi_{b k g}$ adjusted by an additional index $\gamma_{c}$. As the background spectrum is normalized to the AMS-02 datapoint at $E_{0}=11.4 \mathrm{GeV}$, the adjustment is done by multiplication of $\phi_{b k g}$ with $\left(E / E_{0}\right)^{\gamma_{c}}$. Since the CR spectrum is influenced by solar modulation and possibly by diffusive reacceleration and a break of the source spectrum at lower energy, only the AMS-02 data above $15 \mathrm{GeV}$ is used in the fit. The resulting parameters for the modified and unmodified GALPROP with $E_{\text {factor }}=1.3$ are compared to the fit results based on a very fine energy binning $\left(E_{\text {factor }}=1.02\right)$. We minimize $\chi^{2}$ as defined by

$$
\chi^{2}=\sum \frac{\left(\left(\phi_{b k g} \times\left(E / E_{0}\right)^{\gamma_{c}}+\phi_{D M} \times x\right)-\phi_{A M S}\right)^{2}}{\sigma_{A M S}^{2}}
$$

where we sum over the AMS-02 data points $\phi_{A M S}$, with $\sigma_{A M S}$ including statistical and systematic error. To compare with the fine binning fit, for which $x$ and $\gamma_{c}$ are free parameters, we consider three cases: (1) keeping both $x$ and $\gamma_{c}$ fixed to the fine binning fit values and compare $\chi^{2}$; (2) keeping $x$ fixed and comparing the change in $\gamma_{c}$; (3) keeping $\gamma_{c}$ fixed and comparing the change in $x$. As listed in Table 2, the modification reduces the difference in $\chi^{2}$ and in the fit parameters between the results obtained with $E_{\text {factor }}=1.3$ and those with $E_{\text {factor }}=1.02$.

To emphasize that the effect of this modification is comparable to the precision of near future cosmic ray experiments, we study the expected results for CALET on the example of LKP Dark Matter with mass $1 \mathrm{TeV}$, and compare them to the propagation results as shown in Figure 4. The statistical error for the CALET measurement is calculated from the detectors aperture of $1200 \mathrm{~cm}^{2} \cdot \mathrm{sr}$ [7], assuming five years of data-taking and a reconstruction efficiency of $90 \%$. The results for the modified and unmodified version with $E_{\text {factor }}=1.3$ are plotted with the fit parameters obtained with the $E_{\text {factor }}=1.03$ results. 


\begin{tabular}{|l|l|l|l|}
\hline Parameter & $E_{\text {factor }}: 1.02$ & $E_{\text {factor }}: 1.3$ (Unmodified G.) & $E_{\text {factor }}: 1.3$ (Modified G.) \\
\hline$\chi^{2} / \mathrm{NDF}$ & $21.4 / 42($ fit $)$ & $46.6 / 42\left(\gamma_{c}, x\right.$ fixed $)$ & $29.2 / 42\left(\gamma_{c}, x\right.$ fixed $)$ \\
\hline$\gamma_{c}(x=1307)$ & -0.018 & -0.023 & -0.019 \\
\hline$x\left(\gamma_{c}=-0.018\right)$ & 1307 & 1126 & 1250 \\
\hline
\end{tabular}

Table 2: Comparison of $\chi^{2}$ and fit parameters between Modified and Unmodified Galprop.

Since the branching ratio for LKP DM includes $20 \%$ annihilation to the $e^{+} e^{-}$channel, it features a sharp cut-off in the propagated spectrum, corresponding to a sharp peak in the source spectrum, at the mass of the Dark Matter particles. By choosing a large $E_{\text {factor }}$, this peak is widened to the width of the respective energy bin, causing the propagated spectrum to show a difference to a finely binned spectrum near the Dark Matter mass which is not compensated by the described modification to GALPROP. However it is shown that otherwise the results of from the modified version of GALPROP are consistent with those of finer energy binning ( $E_{\text {factor }}: 1.02$ ) within the predicted precision of CALET, while the unmodified version results deviate significantly below the Dark Matter mass.

\section{Summary}

Within the scope of this work a dependence of GALPROP results for electrons on the choice of energy binning was identified and a modification partly compensating it, but causing no increase in computation time implemented. As the memory usage of GALPROP almost directly scales with the number of grid points in space and energy, this modification allows to obtain more precise results on electron propagation with a given computing resource, e.g. in the calculation of nearby astrophysical sources and in studying anisotropy, where a fine spacial grid is required, not allowing to use a fine energy binning simultaneously. Using this modified version of GALPROP to propagate background and DM annihilation cosmic rays consistently, it is found that it can reduce the error induced by the binning on unknown parameters, such as Boost Factor and the background spectrum power law index.

Comparing the propagation results of modified and unmodified version with predicted results for CALET, it is shown that the deviation for coarse energy binning calculations with the unmodified version from results with finer binning are exceeding or at least comparable to the experiment's statistics, while the modification reduces them to a mostly negligible level.

While the modification successfully mitigates the binning dependence on the background power law spectrum, no significant influence on the calculation of the Dark Matter annihilation spectra themselves comes with it. A difference in the propagated Dark Matter spectra remains close to the DM mass for cases with a sharp peak in the injection spectrum. This is most likely attributed to this peak being widened if using a coarse energy binning for the injection spectrum and compensation of this may be a subject of further study. 


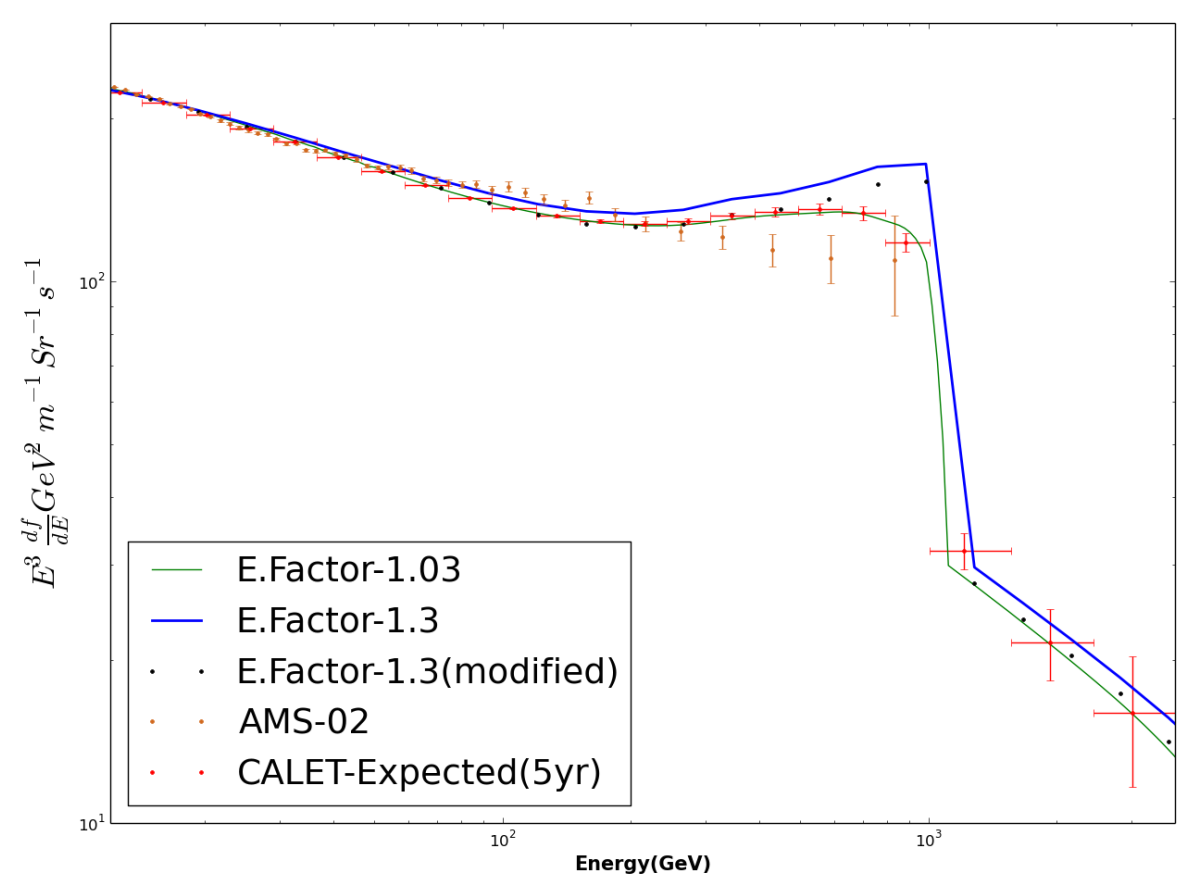

Figure 4: Expected $e^{+}+e^{-}$spectrum by CALET for five years observations (red dots with errorbars, where vertical line signifies statistical error and horizontal line is a measure of bin width for $E_{\text {factor }}: 1.03$ GALPROP simulation) with the simulated spectrum in GALPROP (green line) including LKP Dark Matter (mass $1 \mathrm{TeV}$ ) for an $E_{\text {factor }}: 1.03$ is plotted and compared with a different energy binning $\left(E_{\text {factor }}: 1.3\right)$ calculation (blue line). The effect of modification (black dots) are also shown.

\section{References}

[1] CALET Collaboration, S. Torii. Paper ID 430 in this conference.

[2] http://galprop.stanford.edu/.

[3] A. W. Strong. GALPROP Version 54: Explanatory Supplement(2011).

[4] M. Cirelli, G. Corcella, A. Hektor, G. Hutsi, M. Kadastik, et al. JCAP 1103 (2011) 051.

[5] J. F. Navarro, C. S. Frenk, and S. D. White Astrophys.J. 490 (1997) 493-508.

[6] AMS Collaboration, M. Aguilar et al. Phys. Rev. Lett. 113 (Sep, 2014) 121102.

[7] Y. Akaike, K. Kasahara, and S. Torii in Proceedings of ICRC 2011, vol. 6, p. 371, 2011. 